\title{
Altered cyclophosphamide and thiotepa pharmacokinetics in a patient with moderate renal insufficiency
}

\author{
Corine Ekhart $\cdot$ J. Martijn Kerst $\cdot$ Sjoerd Rodenhuis • \\ Jos H. Beijnen · Alwin D. R. Huitema
}

Received: 22 January 2008 / Accepted: 7 April 2008 / Published online: 23 April 2008

(C) The Author(s) 2008

\begin{abstract}
Purpose We report a patient with renal insufficiency (creatinine clearance, $\mathrm{CL}_{\mathrm{cr}}=38 \mathrm{~mL} / \mathrm{min}$ ) who received high-dose chemotherapy with cyclophosphamide $(1,500$ $\mathrm{mg} / \mathrm{m}^{2}$ day $\left.^{-1}\right)$, thiotepa $\left(120 \mathrm{mg} / \mathrm{m}^{2}\right.$ day $\left.^{-1}\right)$ and carboplatin (AUC $=5 \mathrm{mg} \mathrm{min} / \mathrm{mL} \mathrm{day}^{-1}$ ) for four consecutive days.

Methods Blood samples were collected on day 1 and 3 and plasma levels of cyclophosphamide, its active metabolite 4-hydroxycyclophosphamide, thiotepa, its main metabolite tepa and carboplatin were determined.

Results Pharmacokinetic analyses indicated that the elimination of cyclophosphamide, thiotepa, carboplatin, but especially tepa was strongly reduced in this patient, resulting in increased exposures to these compounds of 67 , 43, 30 and $157 \%$, respectively, compared to a reference population $(n=24)$ receiving similar doses. Exposure to 4-hydroxycyclophosphamide increased $11 \%$.

Conclusion These results suggest that it may not be necessary to alter the dose of cyclophosphamide in patients with moderate renal impairment. However, because high exposures to thiotepa and tepa have been correlated with
\end{abstract}

C. Ekhart $(\varangle) \cdot$ J. H. Beijnen · A. D. R. Huitema Department of Pharmacy and Pharmacology,

The Netherlands Cancer Institute/Slotervaart Hospital, Louwesweg 6, 1066 EC Amsterdam, The Netherlands

e-mail: corine.ekhart@slz.nl

J. M. Kerst $\cdot$ S. Rodenhuis

Department of Medical Oncology,

The Netherlands Cancer Institute/Antoni van

Leeuwenhoek Hospital, Amsterdam, The Netherlands

\section{J. H. Beijnen}

Section of Drug Toxicology, Department of Biomedical Analysis, Faculty of Pharmaceutical Sciences,

Utrecht University, Utrecht, The Netherlands increased toxicity, caution should be applied when administering thiotepa to patients with renal insufficiency.

Keywords Renal insufficiency · Cyclophosphamide . Thiotepa $\cdot$ Pharmacokinetics

\section{Introduction}

Cyclophosphamide and thiotepa are alkylating agents that belong to the most frequently used cytotoxic agents in highdose chemotherapy regimens. A regimen that is commonly used in the Netherlands is the CTC regimen, which consists of cyclophosphamide $\left(6,000 \mathrm{mg} / \mathrm{m}^{2}\right)$, thiotepa $\left(480 \mathrm{mg} / \mathrm{m}^{2}\right)$ and carboplatin $\left(1,600 \mathrm{mg} / \mathrm{m}^{2}\right)$ divided over 4 days administered in short infusions [1].

When using chemotherapeutic agents at the limit of nonhaematological toxicity, there is a substantial risk of adverse outcome. A wide interpatient variability in toxicity of cyclophosphamide, thiotepa and carboplatin has been described, which can be explained in part by the interpatient variability in pharmacokinetics of the respective compounds. Various relations between exposure and toxicity or even efficacy have been identified [2].

Cyclophosphamide is a prodrug that needs to be activated by cytochrome P450 to 4-hydroxycyclophosphamide, which is the main route of metabolism. Both the metabolites and $12-30 \%$ of unchanged parent compound are eliminated in the urine [3]. Thiotepa is rapidly metabolised by cytochrome P450 to tepa, its main and active metabolite. Thiotepa and tepa have similar alkylating properties. Less than $2 \%$ of the administered dose of thiotepa is eliminated unchanged in the urine. Renal elimination of tepa accounts for approximately $11 \%$ of the administered dose $[4,5]$. Carboplatin is a platinum compound, with renal elimination 
accounting for almost all drug elimination. In patients with normal renal function, between 60 and $70 \%$ of an administered dose is excreted in the urine within the first $24 \mathrm{~h}$ of administration [6].

Renal insufficiency is a factor that may complicate drug dosing. When renal function is compromised, drug and metabolites eliminated through the kidneys will retain in the body and may accumulate to toxic levels with repeated dosing. Scarce information is available about the pharmacokinetics of (high dose) cyclophosphamide and thiotepa in patients with renal insufficiency. Earlier studies have described the pharmacokinetics of cyclophosphamide in adults with renal dysfunction receiving either standard dose [7-11] or myeloablative doses of cyclophosphamide $[12,13]$. However, data are controversial. Minimal effects of renal dysfunction upon cyclophosphamide AUC have been reported [8, 11, 12], while others have shown an elevated AUC in those with impaired renal function [9, 10, 13]. A report in an anephric child indicated that off haemodialysis clearance of cyclophosphamide was similar to cyclophosphamide clearance in children with normal renal function [14]. Studies reporting the impact of renal dysfunction upon the metabolites of cyclophosphamide show that plasma alkylating activity was increased in patients with renal failure $[7,10,11]$.

It has been shown that carboplatin clearance is decreased in patients with decreased renal function, resulting in increased toxicity [15]. Dose adjustment of carboplatin is required in these patients. The Calvert formula is widely used for carboplatin dosing based on the glomerular filtration rate [16].

In this report we describe the pharmacokinetics of cyclophosphamide, its active metabolite 4-hydroxycyclophosphamide, thiotepa, its active metabolite tepa and carboplatin in a patient with renal insufficiency.

\section{Patients and methods}

Case

A 40-year-old male patient was diagnosed with Ewing sarcoma. As part of his treatment he received one course of high-dose chemotherapy with autologous peripheral blood progenitor cell transplantation, consisting of cyclophosphamide $\left(1,500 \mathrm{mg} / \mathrm{m}^{2} \mathrm{day}^{-1}\right)$ as 1 -h infusion, immediately followed by carboplatin [dose calculated based on modified Calvert formula: AUC $(\mathrm{mg} \mathrm{min} / \mathrm{mL})=$ Dose $(\mathrm{mg}) /\left(\mathrm{CL}_{\mathrm{cr}}\right.$ $(\mathrm{mL} / \mathrm{min})+25)$ with $5 \mathrm{mg} \mathrm{min} / \mathrm{mL}$ as daily target AUC] as 1 -h infusion and thiotepa $\left(120 \mathrm{mg} / \mathrm{m}^{2} \mathrm{day}^{-1}\right)$ divided over two 30-min infusions $12 \mathrm{~h}$ apart, for four consecutive days. Full details of the CTC regimen have been published previously [17].
The patient had normal cardiac, hepatic, haematopoietic and pulmonary function. Renal function was, however, impaired (calculated creatinine clearance $38 \mathrm{~mL} / \mathrm{min}$, using the Cockcroft-Gault equation [18]).

\section{Pharmacokinetic analysis}

For pharmacokinetic analyses, blood samples were collected from a double lumen intravenous catheter inserted in a subclavian vein. Samples were collected on day 1 and 3 prior to the start of the infusions, at $30 \mathrm{~min}$ after the start of cyclophosphamide infusion $(t=30)$ and $t=60$ (end of cyclophosphamide infusion), 90, 120 (end of carboplatin infusion), 150 (end of thiotepa infusion), 180, 210, 285, 390 and $660 \mathrm{~min}$. Samples were processed as described previously [19-21]. Analytical methods used for the determination of plasma concentrations of cyclophosphamide, 4-hydroxycyclophosphamide, thiotepa, tepa and carboplatin have been reported previously [19-21].

Population pharmacokinetic models of carboplatin, cyclophosphamide (and its metabolite 4-hydroxycyclophosphamide) and thiotepa (and its metabolite tepa) were used for calculating the pharmacokinetic parameters of all compounds using Bayesian analysis [2, 22]. The pharmacokinetic parameters of the different compounds in this patient were compared with the respective median values in a reference population of patients $(n=24)$ who also received CTC and were dosed (except for carboplatin, reference population received $400 \mathrm{mg} / \mathrm{m}^{2}$ day $^{-1}$ ) and sampled as described above. Complete pharmacokinetic profiles of the reference population were available of day 1 and day 3 or 4. Exposure was defined as the cumulative exposure of the 4-day course extrapolated to infinity. The protocol was approved by the Committee on the Medical Ethics of the Netherlands Cancer Institute and written informed consent was obtained from the patient.

\section{Results}

Administration of cyclophosphamide, thiotepa and carboplatin in this patient led to increased exposures to all compounds. Table 1 shows the median cumulative exposures following one course in both the population and the patient, the latter based on the pharmacokinetics obtained on the first day of the 4-day course. Figure 1 shows the plasma concentration-time data of the patient versus the typical plasma concentration-time curve of the reference population [2, 22].

Comparing pharmacokinetic parameters of cyclophosphamide, thiotepa and carboplatin in this patient with those in the reference population, it appeared that the renal clearance of cyclophosphamide and thiotepa decreased 
Table 1 Overall exposure (expressed as AUC) to the different compounds and their metabolites during the course, if no dose adjustment would have been done

\begin{tabular}{llcccc}
\hline Compound & AUC units & AUC patient & Median AUC reference population & 2.5-97.5\% range & Deviation (\%) \\
\hline Cyclophosphamide & $\mathrm{uM} \mathrm{h}$ & 11,467 & 6,871 & $4,355-10,664$ & 67 \\
4-Hydroxycyclophosphamide & $\mathrm{uM} \mathrm{h}$ & 157 & 142 & $105-186$ & 11 \\
Thiotepa & $\mathrm{uM} \mathrm{h}$ & 185 & 129 & $81-201$ & $108-427$ \\
Tepa & $\mathrm{uM} \mathrm{h}$ & 648 & 252 & $222-584$ & 157 \\
Thiotepa and tepa & $\mathrm{uM} \mathrm{h}$ & 833 & 384 & 30 \\
Carboplatin & $\mathrm{mg} \mathrm{min} / \mathrm{mL}$ & 26 & $20^{\mathrm{a}}$ & 117 \\
\hline
\end{tabular}

a Target AUC value, reference population received carboplatin dose of $400 \mathrm{mg} / \mathrm{m}^{2}$ day $^{-1}$

Fig. 1 Concentration-time curves of a cyclophosphamide (CP), b 4-hydroxycyclophosphamide (4OHCP), $\mathbf{c}$ thiotepa (TT), d tepa (T) and e carboplatin (CA) with triangle representing the patient and filled square the typical plasma concentration-time curve of the reference population
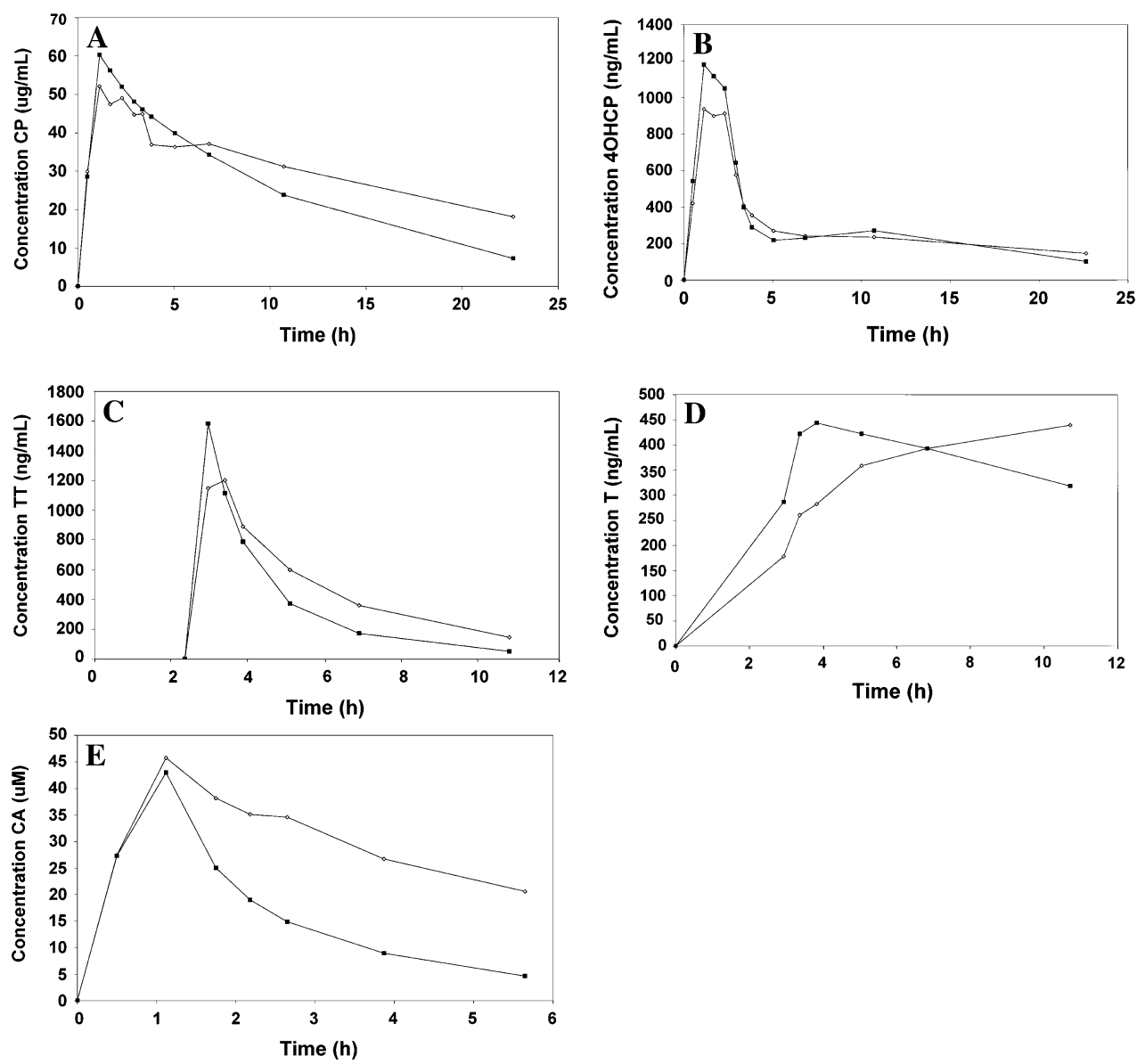

in the patient compared to the reference population with 28 and $36 \%$, respectively (2.1 vs. $2.9 \mathrm{~L} / \mathrm{h}$ and 15.9 vs. $24.9 \mathrm{~L} / \mathrm{h}$ ). This resulted in an increased exposure to cyclophosphamide and thiotepa of 67 and 43\%, respectively. Exposure to 4-hydroxycyclophosphamide was only moderately increased, due to a minor decrease in elimination of 4-hydroxycyclophosphamide $(7 \%)$ and a minor increase in metabolic fraction (11\%), compared to the reference population. Exposure to tepa, however, increased dramatically (157\%), mainly due to a decreased elimination rate constant of tepa $\left(0.23 \mathrm{~h}^{-1}\right.$ in the patient vs. $0.47 \mathrm{~h}^{-1}$ in the reference population). Although carboplatin was dosed based on creatinine clearance the exposure was still $30 \%$ above the target value (26 vs. $20 \mathrm{mg} \mathrm{min} / \mathrm{mL}$ ).

Based on the pharmacokinetic analyses of day 1 of the course, the doses of cyclophosphamide, thiotepa and carboplatin were reduced on days 3 and 4 to reach a predefined target exposure (the median exposure in a reference population receiving similar doses) [23]. The cyclophosphamide dose was reduced from 2,955 to $2,550 \mathrm{mg} /$ day, the thiotepa dose from 236 to $162 \mathrm{mg} /$ day and the carboplatin dose from 315 to $275 \mathrm{mg} /$ day. This resulted in an exposure closer to 
the target exposure. Combined exposure to thiotepa and tepa, however, was still $75 \%$ above the target. Renal function remained the same during the 4 days of chemotherapy.

The patient experienced grade 3 mucositis and diarrhoea. Furthermore, grade 2 aspartate aminotransferase (ASAT) and bilirubin toxicity was observed. In the reference population three patients experienced grade 3 mucositis $(12.5 \%)$, no patients had grade 3 diarrhoea, six patients experienced grade 2 ASAT toxicity (25\%) and one patient had grade 2 bilirubin toxicity (4.2\%). On day 43 post-transplantation the patient died due to acute respiratory distress syndrome.

\section{Discussion}

Renal insufficiency alters the pharmacokinetics of drugs. If drugs or their active metabolites are excreted by the kidneys they can accumulate in patients with renal insufficiency. In the patient reported here, increased exposures to cyclophosphamide, 4-hydroxycyclophosphamide, thiotepa, tepa and carboplatin were observed.

The patient described here experienced excessive exposure to tepa. Renal elimination of thiotepa accounts for only a small part of its elimination, for tepa, apparently, renal elimination is an important route. Toxicities associated with thiotepa treatment are mainly mucositis and central nervous system toxicity. The most frequently affected organs being the liver and gastrointestinal tract, resulting in nausea, vomiting and diarrhoea [4]. Moreover, a relation between ASAT toxicity and thiotepa AUC has been demonstrated [2]. Indeed, this patient experienced severe mucositis and diarrhoea and grade 2 ASAT toxicity, probably due to excessive exposure to thiotepa and tepa.

Clearance of cyclophosphamide was reduced, which resulted in an increase in cyclophosphamide exposure. Since cyclophosphamide itself is not active, increases in exposure to the active metabolites are more important for the interpretation of the clinical relevance of this effect. The exposure to 4-hydroxycylophosphamide was only moderately increased, indicating no need to adjust the dose of cyclophosphamide in patients with moderate renal insufficiency. Our data are in agreement with the data of Juma et al. [10] who reported a lower clearance $(-17 \%)$ and an increase in half-life $(+24 \%)$ of cyclophosphamide in five patients with moderate to severe renal insufficiency (creatinine clearances $18-51 \mathrm{~mL} / \mathrm{min}$ ) compared to eight matched controls with normal renal function. Similar results were reported by Haubitz et al. [9] who found a lower clearance $(-28 \%)$ of cyclophosphamide in patients with creatinine clearances of $25-50 \mathrm{~mL} / \mathrm{min}$, which resulted in an increase in systemic drug exposure of $38 \%$. Mouridsen et al. [11] showed that the biotransformation rate is unaffected in patients with renal impairment. Bramwell et al. [8] could not demonstrate a correlation between renal function and clearance of cyclophosphamide or its alkylating metabolites due to the large inter-individual variability in cyclophosphamide break-down seen in that study. Further data regarding the impact of renal dysfunction upon the metabolites of cyclophosphamide are scarce. One study showed that total alkylating activity as measured by the nitrobenzylpyridine (NBP) reaction was significantly increased in renal failure [10]. However, this NBP reaction is highly non-specific and variable [24]. Therefore, no firm conclusions could be attributed to this result. A report about cyclophosphamide disposition in an anephric child showed that the exposure to 4-hydroxycylophosphamide off haemodialysis was in the same range as our results [14]. Clinically relevant changes in cyclophosphamide pharmacology due to alterations in renal function have not been demonstrated.

For carboplatin it is known that renal insufficiency causes increased exposure to carboplatin. Therefore, a priori dose adjustments of carboplatin are performed. Although this patient had an adjusted dose of carboplatin based on his creatinine clearance, exposure to carboplatin was still $30 \%$ above the target exposure. Substitution of the glomerular filtration rate in the Calvert formula by an estimate of creatinine clearance calculated using the Cockcroft-Gault equation led to an overestimation of the carboplatin clearance in this patient. Froissart et al. [25] showed that the use of the Cockcroft-Gault equation can lead to overestimation of the glomerular filtration rate in patients with GFR $<60 \mathrm{~mL} / \mathrm{min}$.

In conclusion, this case report demonstrates the pharmacokinetic disposition of cyclophosphamide, 4-hydroxycyclophosphamide, thiotepa, tepa and carboplatin in a patient with reduced renal function. Renal insufficiency in this patient resulted in a high exposure to thiotepa and especially tepa and is, therefore, of potential clinical importance. Additional pharmacokinetic and pharmacodynamic data are warranted in future studies to provide more accurate dosage recommendations for thiotepa in these patients.

Acknowledgment This work was supported with a grant from the Dutch Cancer Society (project NKI 2005-3418).

Open Access This article is distributed under the terms of the Creative Commons Attribution Noncommercial License which permits any noncommercial use, distribution, and reproduction in any medium, provided the original author(s) and source are credited.

\section{References}

1. Rodenhuis S, Richel DJ, van der WE, Schornagel JH, Baars JW, Koning CC et al (1998) Randomised trial of high-dose chemo- 
therapy and haemopoietic progenitor-cell support in operable breast cancer with extensive axillary lymph-node involvement. Lancet 352(9127):515-521

2. Huitema ADR, Spaander M, Mathot RAA, Tibben MM, Holtkamp MJ, Beijnen JH et al (2002) Relationship between exposure and toxicity in high-dose chemotherapy with cyclophosphamide, thiotepa and carboplatin. Ann Oncol 13(3):374-384

3. de Jonge ME, Huitema ADR, Rodenhuis S, Beijnen JH (2005) Clinical pharmacokinetics of cyclophosphamide. Clin Pharmacokinet 44(11):1135-1164

4. van Maanen MJ, Smeets CJ, Beijnen JH (2000) Chemistry, pharmacology and pharmacokinetics of N, N', N" -triethylenethiophosphoramide (ThioTEPA). Cancer Treat Rev 26(4):257-268

5. Huitema ADR, Smits KD, Mathot RA, Schellens JH, Rodenhuis S, Beijnen JH (2000) The clinical pharmacology of alkylating agents in high-dose chemotherapy. Anticancer Drugs 11(7):515-533

6. van der Vijgh WJ (1991) Clinical pharmacokinetics of carboplatin. Clin Pharmacokinet 21(4):242-261

7. Bagley CM Jr, Bostick FW, DeVita VT Jr. Clinical pharmacology of cyclophosphamide. Cancer Res 1973; 33(2):226-233

8. Bramwell V, Calvert RT, Edwards G, Scarffe H, Crowther D (1979) The disposition of cyclophosphamide in a group of myeloma patients. Cancer Chemother Pharmacol 3(4):253-259

9. Haubitz M, Bohnenstengel F, Brunkhorst R, Schwab M, Hofmann U, Busse D (2002) Cyclophosphamide pharmacokinetics and dose requirements in patients with renal insufficiency. Kidney Int 61(4):1495-1501

10. Juma FD, Rogers HJ, Trounce JR (1981) Effect of renal insufficiency on the pharmacokinetics of cyclophosphamide and some of its metabolites. Eur J Clin Pharmacol 19(6):443-451

11. Mouridsen HT, Jacobsen E (1975) Pharmacokinetics of cyclophosphamide in renal failure. Acta Pharmacol Toxicol (Copenh) 36(Suppl 5):409-414

12. Bischoff ME, Blau W, Wagner T, Wagenmann W, Dorner O, Basara N et al (1998) Total body irradiation and cyclophosphamide is a conditioning regimen for unrelated bone marrow transplantation in a patient with chronic myelogenous leukemia and renal failure on hemodialysis. Bone Marrow Transplant 22(6):591-593

13. Perry JJ, Fleming RA, Rocco MV, Petros WP, Bleyer AJ, Radford JE Jr et al (1999) Administration and pharmacokinetics of highdose cyclophosphamide with hemodialysis support for allogeneic bone marrow transplantation in acute leukemia and end-stage renal disease. Bone Marrow Transplant 23(8):839-842

14. McCune JS, Adams D, Homans AC, Guillot A, Iacono L, Stewart CF (2006) Cyclophosphamide disposition in an anephric child. Pediatr Blood Cancer 46(1):99-104
15. Egorin MJ, Van Echo DA, Tipping SJ, Olman EA, Whitacre MY, Thompson BW et al (1984) Pharmacokinetics and dosage reduction of cis-diammine (1, 1-cyclobutanedicarboxylato) platinum in patients with impaired renal function. Cancer Res 44(11):54325438

16. Calvert AH, Newell DR, Gumbrell LA, O'Reilly S, Burnell M, Boxall FE et al (1989) Carboplatin dosage: prospective evaluation of a simple formula based on renal function. J Clin Oncol 7(11):1748-1756

17. Rodenhuis S, Bontenbal M, Beex LV, Wagstaff J, Richel DJ, Nooij MA et al (2003) High-dose chemotherapy with hematopoietic stem-cell rescue for high-risk breast cancer. N Engl J Med 349(1):7-16

18. Cockcroft DW, Gault MH (1976) Prediction of creatinine clearance from serum creatinine. Nephron 16(1):31-41

19. Ekhart C, Gebretensae A, Rosing H, Rodenhuis S, Beijnen JH, Huitema ADR (2007) Simultaneous quantification of cyclophosphamide and its active metabolite 4-hydroxycyclophosphamide in human plasma by high-performance liquid chromatography coupled with electrospray ionization tandem mass spectrometry (LCMS/MS). J Chromatogr B Analyt Technol Biomed Life Sci 854(12):345-349

20. Huitema ADR, Tibben MM, Kerbusch T, Zwikker JW, Rodenhuis S, Beijnen JH (1998) Simultaneous determination of N, N', N"-triethylenethiophosphoramide, cyclophosphamide and some of their metabolites in plasma using capillary gas chromatography. J Chromatogr B Biomed Sci Appl 716(1-2):177-186

21. Van Warmerdam LJC, Van Tellingen O, Maes RAA, Beijnen J (1995) Validated method for the determination of carboplatin in biological fluids by Zeeman atomic absorption spectrometry. Fresenius' journal of analytical chemistry 351:777-781

22. de Jonge ME, Huitema ADR, Rodenhuis S, Beijnen JH (2004) Integrated population pharmacokinetic model of both cyclophosphamide and thiotepa suggesting a mutual drug-drug interaction. J Pharmacokinet Pharmacodyn 31(2):135-156

23. de Jonge ME, Huitema ADR, Tukker AC, van Dam SM, Rodenhuis S, Beijnen JH (2005) Accuracy, feasibility, and clinical impact of prospective Bayesian pharmacokinetically guided dosing of cyclophosphamide, thiotepa, and carboplatin in high-dose chemotherapy. Clin Cancer Res 11(1):273-283

24. Lewis LD (1991) Ifosfamide pharmacokinetics. Invest New Drugs 9(4):305-311

25. Froissart M, Rossert J, Jacquot C, Paillard M, Houillier P (2005) Predictive performance of the modification of diet in renal disease and Cockcroft-Gault equations for estimating renal function. J Am Soc Nephrol 16(3):763-773 